Article

\title{
Spatial Access to Medical Services in the Underdeveloped Region of Northwestern China: A Case Study in Lanzhou City
}

\author{
Wantong Xu, Fuguang Zhang, Biao Zeng * ${ }^{\mathbb{D}}$, Taibao Yang and Hui Wang \\ College of Earth and Environmental Sciences, Lanzhou University, Lanzhou 730000, China; \\ xuwantong@msn.cn (W.X.); zhangfg15@lzu.edu.cn (F.Z.); yangtb@lzu.edu.cn (T.Y.); \\ huiwang2018@lzu.edu.cn (H.W.) \\ * Correspondence: zengb@lzu.edu.cn
}

Received: 14 October 2019; Accepted: 26 November 2019; Published: 29 November 2019

check for updates

\begin{abstract}
Research on the spatial access to medical services has become a hot topic in recent years. The representative provincial capital in the underdeveloped region of northwestern China, Lanzhou, was selected for the research area. In this paper, methods such as the two-step floating catchment area and ArcGIS network analysis are used to analyze the geographic spatial accessibility of medical services and differences of spatial access between urban and rural areas in Lanzhou city. The results show that 1. Areas in General and below grade of accessibility account for most of Lanzhou city. Therefore, the spatial accessibility of whole Lanzhou city is comparatively unsatisfactory. It shows a tendency of the north region to be worse than south region, and areas in parts of the main districts, parts of HG, and other tiny minority show Great grade of accessibility, and most of other areas distribute in poorer grade of accessibility, especially the surrounding mountainous region. 2. There are obvious differences in accessibility between urban and rural regions, mainly reflected in that residents and areas are basically districted in the Great and Good grade in the urban region, while almost $75 \%$ of the population and $35 \%$ of the areas are distributed in the Great and Good grade, and nearly $25 \%$ of the residents and $65 \%$ of the areas are still in the unsatisfactory accessibility grade in the rural region. According to the results, the researchers put forward corresponding suggestions to improve the current situation, which are roughly as follows: The construction of hospitals and primary health care should be strengthened in the rural region, especially the construction of township health centers and health stations. High-class hospitals are needed in Honggu district, Yongdeng county, Yuzhong county, and Gaolan county, while the medical capacity and facility grade of existing hospitals should be improved to satisfy the demand of local residents. Meanwhile, rural areas should strengthen the construction of road networks.
\end{abstract}

Keywords: two-step floating catchment area; network analyst; spatial access to medical services; urban-rural disparities; undeveloped region of northwestern China

\section{Introduction}

It is one of the main imperatives of health care geography to study the reasonable geographical allocation of health care institutions and facilities, which involves analyzing the spatial allocation and functions of medical service institutions, including geographic locations and spatial distribution of them, and the number of medical staff and beds. Geography of health care studies the relationship between the spatial distribution of medical services and the demand of an area's residents, the reasons for destroying the balance and its spatial distribution pattern, and the condition of maintaining the balance. Meanwhile, it describes and explains the characteristic of providing services, the usage type 
of different services, and the relationship between types of service and health outcomes in different geographic scale [1]. In the study of health care geography, combining the functions of a medical service institution and the concept of accessibility can intuitively analyze the medical service in space, as spatial accessibility is an effective measurement standard for the service capacity of medical service facilities.

Access has both spatial and aspatial dimensions [2]. Spatial access analyzes the importance of spatial separation between supply and demand as a barrier or a facilitator, and aspatial access focuses on non-geographical barriers or facilitators [3,4]. In this paper, spatial access is the main research direction. Spatial access consists of two parts, which are proximity and availability [5]. The former describes the proximity (it can be distance or time) of the demand to medical services in space. And the latter signifies the size of the demand competing for available services. Both of them need to be measured together when defining spatial access [6]. The physical mechanism of spatial access interprets as compatibility between population area, transport network, and distribution of medical institutions, which reflects the condition of spatial access. High and low density of different factors makes the spatial accessibility appear various complexion. Guagliardo [7] and Luo et al. [3] selected a facility's number of beds, number of medical staff, transportation network, and population density as factors by which to evaluate the spatial access to medical services. Since then, significant breakthroughs have been made in research on the spatial accessibility of medical services, both in terms of technology and content. At present, the main research contents about the spatial accessibility of medical services roughly include: The influence of geographic environment on the spatial distribution of a particular disease [8]; research on accessibility model [6,9]; disparity of spatial accessibility in different traffic conditions [10,11]; and medical inequality $[12,13]$. And in this paper, the main study refers to spatial accessibility and its inequality between urban and rural regions.

Plenty of methods have been put forward by scholars in order to study the above-mentioned research contents. The main measurement approaches of spatial access to medical services comprises ratio method, Nearest Distance method, method based on opportunity accumulation such as floating catchment area (FCA) and two-step floating catchment area (2SFCA), and method based on spatial interaction such as potential model, Huff model, and Kernel Density Method. Ratio method cannot reflect the change of accessibility in the research unit, and it does not consider the spatial barriers between the supply and the demand. Nearest Distance method ignores the quantity and quality of services. The travel friction coefficient of potential model et al. is difficult to determine, and the models are hard to comprehend. 2SFCA synthetically considers the facilities, demanders, and space barriers between the supply and demand and other factors. The limitation of traditional 2SFCA is lack of the consideration of the distance attenuation [14]. In this paper, a modified 2SFCA was selected as the main approach for analyzing, and the distance attenuation is also taken into account, which considers the impact of increased spatial distance in the search area on residents' access to medical services. All in all, 2SFCA is a relatively comprehensive, reasonable, and reliable measurement approach of spatial access to medical service.

Classification of health care system plays a decisive role in spatial access, and different search radii are set for different class of medical institutions in this research. By means of understanding of the health care system network in China, it could strengthen comprehension of analysis in spatial access. Health care institutions in China can be classified into four categories: Hospitals, primary health care institutions, specialized public health institutions, and other institutions [15]. Hospitals are segmented into three tiers, respectively tertiary hospital, secondary hospital, and primary hospital. The primary health care system consists of rural and urban parts. The rural system comprises village health stations and township health centers, while the urban part comprises clinics and community health centers.

Research on the spatial access to medical services has mostly concentrated on the southern region and eastern coastal area of China, like what Chen et al. [16] compared and analyzed the impact of three groups of different traffic conditions on emergency medical services in Guangzhou. Song et al. [14] utilized the improved potential model to measure the medical spatial accessibility of Rudong County in Jiangsu province and judge the area's lacking medical services. Gao et al. [17] used the improved 
2SFCA method to study the spatial accessibility of medical facilities in Shenyang. Wang et al. [15] studied the spatial accessibility of primary health care in Sichuan province based on the Gini coefficient. Thus, for the northwestern region of domestic limited accessibility of medical services research, and with Lanzhou city being the representative provincial capital in the underdeveloped northwestern region of China, choosing it as the study area can preliminarily identify and illustrate the rationality of the distribution of medical equipment, the equity of medical resources, and the disparity between urban and rural in this region, in order to provide foundation for further study. Meanwhile, the combination with modern research technology of medical service institutions and geography can make the medical resources reasonably allocated and provide a scientific basis for the planning of public service facilities in urban planning.

In this paper, spatial access to medical services is analyzed by 2SFCA and other approaches, which is aimed at explaining whether the distribution of medical facilities is reasonable, whether supply and demand are balanced, whether there is spatial compatibility between residential areas and infrastructure, and the degree of medical resource disparity between urban and rural regions.

\section{Materials and Methods}

\subsection{Study Area}

Lanzhou, located in northwest China and central Gansu province, is the province capital, an important industrial base, and a comprehensive transportation hub in northwest China. It is also one of the most important central and representative cities in northwest China, and the total area of city is $13085.6 \mathrm{~km}^{2}$. Topographically, Lanzhou is long and narrow, with complex and varied land formations. The south and west of Lanzhou topography are at a higher elevation, while the northeast is low. The administrative division of the study area is shown in Figure 1. By the end of 2017, Lanzhou had a permanent population of almost 4 million, including $70 \%$ in urban region and $30 \%$ in rural region, respectively. The administrative division of Lanzhou is spread over three counties and five districts: Yuzhong County (YZ), Yongdeng County (YD), Gaolan County (GL), Chengguan District (CG), Anning District (AN), Xigu District (XG), Qilihe District (QLH), and Honggu District (HG). Among them, CG, XG, AN, and QLH are the main districts.

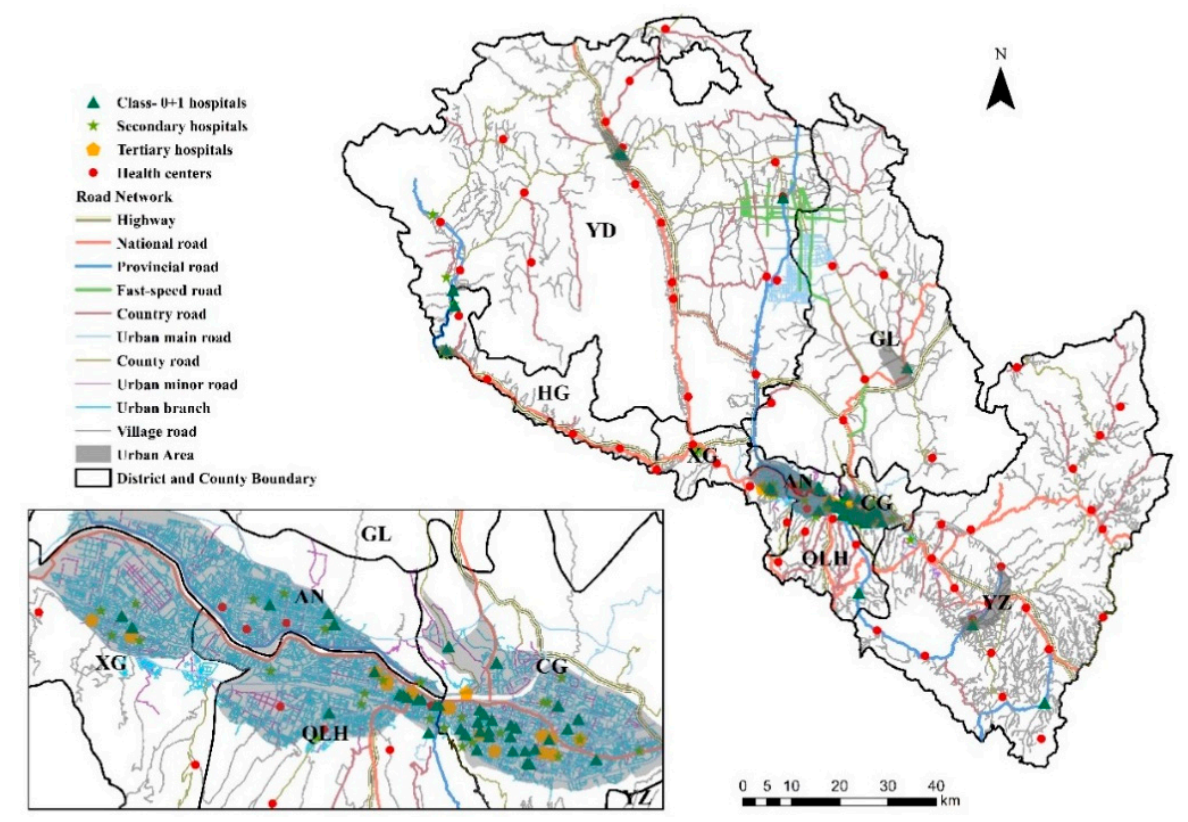

Figure 1. Fundamental information in Lanzhou city. 


\subsection{Data}

The spatial analysis of medical services in Lanzhou mainly involves supply points, demand points, and the distance between them. After concretizing, the main data includes resident points, medical institution points, and transportation networks. The medical institutions studied include hospitals, health centers, clinics, and village clinics.

\subsubsection{Population}

In this study, in order to obtain accurate spatial distribution of population, Worldpop [18] population raster data in 2015 is applied because of its high resolution and accurate spatially-detailed demographic data. The central points of the 1000-meter spatial resolution raster, transferred from the WorldPop population density raster, were selected as the population analysis points. The specific processing method of population points was as follows: First, cutting the WorldPop 100-meter spatial resolution raster data according to the Gansu province boundary, and then resampling the raster into a 1000-meter spatial resolution raster. In the process of resampling, pixel data was adopted by method of sum superposition. After that, researchers cut the 1000-meter spatial resolution raster by means of Lanzhou city boundary. Finally, the data was converted to raster points for subsequent data analysis.

\subsubsection{Medical Institutions (MI)}

Hospitals in Lanzhou city include unrated general/specialized hospitals, primary general/ specialized hospitals, secondary general/specialized hospitals, and tertiary general/specialized hospitals, a total of 112 hospital facilities. Among them, unrated general/specialized hospitals and primary general/specialized hospitals were put into one group for data calculation, because they have nearly identical scales and service capacity, and the following are collectively referred to as class $0+1$ hospitals. Source: Each of medical institutions and data of Lanzhou Statistics Department in 2017.

Data of primary health care in Lanzhou city are from Baidu 2017 Points of Interest, mainly including township health centers, village health stations, polyclinics, and specialized clinics. According to statistics, there are 761 primary health care facilities total in Lanzhou, including 66 township health centers, 314 village health clinics, and 314 polyclinics/specialized clinics. The general situation of hospitals and health centers in Lanzhou is shown in Table 1, and the distribution of medical institution is shown in Figure 1.

Table 1. General situation of medical institution in Lanzhou city.

\begin{tabular}{cccccc}
\hline & $\begin{array}{c}\text { Number of } \\
\text { Class 0 + 1 } \\
\text { Hospitals }\end{array}$ & $\begin{array}{c}\text { Number of } \\
\text { Secondary } \\
\text { Hospitals }\end{array}$ & $\begin{array}{c}\text { Number of } \\
\text { Tertiary } \\
\text { Hospitals }\end{array}$ & $\begin{array}{c}\text { Number of } \\
\text { Hospital Beds } \\
\text { (Per Mille) }\end{array}$ & $\begin{array}{c}\text { Number of } \\
\text { Health-Centers }\end{array}$ \\
\hline CG & 25 & 12 & 12 & 9.95 & 0 \\
AN & 3 & 5 & 0 & 2.46 & 1 \\
XG & 2 & 7 & 2 & 4.28 & 5 \\
QLH & 11 & 8 & 4 & 10.98 & 5 \\
HG & 2 & 5 & 0 & 9.38 & 3 \\
GL & 1 & 1 & 0 & 3.29 & 7 \\
YD & 5 & 4 & 0 & 3.5 & 18 \\
YZ & 1 & 1 & 0 & 3.72 & 21 \\
\hline
\end{tabular}




\subsubsection{Road Network}

Carriageway was drawn based on Google Earth and the open street map, which is used to calculate the cost of transportation from population points to medical institutions. The main types of roadways are: Highways, national roads, provincial roads, fast-speed roads, country roads, urban main roads, county roads, urban minor roads, urban branches, and village roads. Speed limits of the roads are respectively assigned according to government's Department of Transport. The assignments of road networks are shown in Table 2, and the distribution of road networks is shown in Figure 1.

Table 2. Speed limits of road network in Lanzhou city.

\begin{tabular}{|c|c|}
\hline Road Type & Speed $\left(\mathrm{km} \cdot \mathrm{h}^{-1}\right)$ \\
\hline Highway & 100 \\
\hline National road & 70 \\
\hline Provincial road & 70 \\
\hline Fast-speed road & 80 \\
\hline Country road & 40 \\
\hline Urban main road & 50 \\
\hline County road & 40 \\
\hline Urban minor road & 40 \\
\hline Urban branch & 30 \\
\hline Village road & 30 \\
\hline
\end{tabular}

\subsection{Network Analyst of ArcGIS}

ArcGIS network analysis is a network-based spatial analysis extension module for route selection, which can use geographic networks to solve various problems, and perform many network-based tasks. In this paper, after topology analysis processing of the road networks, a network dataset was constructed to calculate the O-D Cost Matrix between population points and medical institutions, which was used to solve the accessibility of medical services. The analysis functions of the Closet Facilities and Service Area under the network analysis module were also applied.

\section{4. $2 S F C A$}

The two-step floating catchment area method (2SFCA), first proposed by Radke and Mu [19], is widely used in the research of communal facilities such as those used for medical care, green land, and old-age care. As it considers both the demand side and the supply side, the result is more holistic than the floating catchment area method. The first step of 2SFCA is to take the supply points as the center, search the demand points in the corresponding range according to the set search radius, and obtain the supply-demand ratio.

The formula is:

$$
V_{j}=\frac{S_{j}}{\sum_{k \in d_{k j} \leq d_{0}} P_{k}}
$$

In Formula (1), $V_{j}$ is ratio of the capacity of supply $j$, which in this paper is measured by the ratio of the number of beds to population served within search radius $d_{0} . d_{k j}$ is the travel time between demand $k$ and supply $j . P_{k}$ represents the demand scale of demand point $k$, which is measured by the population of each demand point.

The second step is to take the demand point as the center, according to the supply points within the search range of the set search radius, and then sum the supply-to-demand ratio to calculate the accessibility value $A_{i}$.

This formula is:

$$
A_{i}=\sum_{j \in d_{i j} \leq d_{0}} V_{j}
$$


In Formula (2), $d_{i j}$ is the travel time between demand $i$ and supply $j . A_{i}$ represents the accessibility score of demand $i$ calculated according to the 2SFCA. The actual meaning is the facility resources available by each demander of demand $i$.

\subsubsection{Modified 2SFCA (M2SFCA)}

Delamater [20] indicated that there is an implicit assumption both in 2SFCA and its existing improved forms; that is, all the facilities supply points in space can obtain optimal configuration to satisfy the demand of the system, which is manifested as that which is based on the evaluation result of the existing 2SFCA model group, totaling up the accessibility score of each demand point multiplied by its demand scale and identically equal to the supply point scale, which means that all the supply resources would be fully used.

This assumption is difficult to illustrate in real life, however. Therefore, Delamater [20] put forward an important extension form, Modified 2SFCA, which allows sub-optimal spatial configurations of facilities to exist; that is, some facility resources cannot be allocated to the demand points, and thus cannot be used effectively. The aforementioned weighted sum of the demand scale of accessibility score has a certain amount of losses compared with the total supply scale. This problem can be summarized as follows: Although 2SFCA and its existing improvement forms measure the concept of accessibility well, they lack consideration of the usability of facility resources, and the M2SFCA realizes the measurement of usability on the basis of accessibility.

The formula of M2SFCA model is:

$$
A_{i}=\sum_{j=1}^{n} \frac{S_{j} f\left(d_{i j}\right) f\left(d_{i j}\right)}{\sum_{k=1}^{m} D_{k} f\left(d_{k j}\right)}
$$

In Formula (3), $f\left(d_{i j}\right)$ is the generalized distance attenuation function, and other variates have the same meaning as Formulas (1) and (2).

\subsubsection{The Distance Attenuation Function}

The generalized distance attenuation function formula is:

$$
f\left(d_{i j}\right)=\left\{\begin{aligned}
g\left(d_{i j}\right), d_{i j} & \leq d_{0} \\
0, d_{i j} & >d_{0}
\end{aligned}\right.
$$

In Formula (4), $g\left(d_{i j}\right)$ represents the distance attenuation function within the search radius $d_{0}$.

In previous studies, there are many functions that can be plugged into the distance attenuation function [21]. Likewise, in this paper, the Gaussian function was chosen to plug into M2SFCA model. The concept of Gaussian-type 2SFCA first developed by Dai [22] who used Gaussian function as the distance attenuation function of 2SFCA. The accessibility attenuation rate of Gaussian-type distance attenuation function would speed up and then slow down with the increase of distance. Gaussian-type 2SFCA requires high accuracy of demand points. On the premise of ensuring accurate data of demand points, the selection of Gaussian distance attenuation function can make the results more accurate.

This formula is:

$$
g\left(d_{i j}\right)=\frac{e^{-\frac{1}{2} \times\left(\frac{d_{i j}}{d_{0}}\right)^{2}-e^{-\frac{1}{2}}}}{1-e^{-\frac{1}{2}}}, d_{i j} \leq d_{0}
$$

\subsubsection{Search Radius $d_{0}$}

One of the disadvantages of 2SFCA is that it adopts a fixed search radius, which is uniform for all supply and demand points, and does not agree with actual states. In light of this problem, scholars have put forward their own opinions [23,24]. In present research, there are four kinds of expansion models of 2SFCA for search radius [21], which are: Variable 2SFCA (V2SFCA), originally proposed by 
Luo [23]; Dynamic 2SFCA (D2SFCA), first proposed by McGrail [25]; Multi Catchment Sized 2SFCA (MCS2SFCA), developed by Tao [26]; and Nearest-Neighbor 2SFCA (N2SFCA), first put forward by Jamtsho [27]. Combined with the actual situation and the actual research significance of this paper's study area, the setting of a search radius according to the standard of hospital classification was used in this paper. That is, a different search radius was set to each hospital class. Township health centers were set to $30 \mathrm{~min}$; unrated hospitals were set to $60 \mathrm{~min}$; class- 1 hospitals were set to $90 \mathrm{~min}$; secondary hospitals were set to $120 \mathrm{~min}$; and tertiary hospitals were set to $150 \mathrm{~min}$.

The steps are as follows: Firstly, basic data such as road network, number of population, and number of beds in medical facilities had been preprocessed. Then, set different search radius $d_{0}$ based on the class of hospital. Secondly, substitute the set $d_{0}$ into the distance attenuation function Formula (5) for calculation. Finally, the number of population, the number of beds, and distance attenuation functions should be substituted into M2SFCA Formula (3) for calculation.

\section{Results}

\subsection{Differences in Spatial Accessibility of Medical Services}

Differences in spatial accessibility cannot be simply defined by a straight line between medical facilities and population points in space. Owing to the distribution of road network, there would be some impedance in actual research. Before calculating the spatial accessibility of medical services, the time impedance of the population points to the nearest medical institutions calculated would provide a basis and clarification for the analysis of accessibility differences in space.

The Closet Facilities tool in the network analyst module is used to solve the time from population points to the nearest primary health care facility, and the time from population points to the nearest hospital, respectively. According to Table 3, reaching the nearest primary health care takes $40.48 \mathrm{~min}$ on average, and $78 \%$ of the population can obtain medical services from township health centers in $15 \mathrm{~min}$, while only $23.2 \%$ of the whole area is covered. In a 15 to $30 \mathrm{~min}$ timeframe, $26.6 \%$ of the region can obtain primary medical services, but only $12.3 \%$ of the population is radiated, which shows that this kind of situation mostly occurs in suburbs and rural area with less population. In the 30 to $60 \mathrm{~min}$ timeframe, $32.5 \%$ of the area is covered-the largest percentage of area covered-but it only contains $7.2 \%$ of the population, which is the same with the previous timeframe. Reaching the nearest hospital takes $64 \mathrm{~min}$, and more than half the population (69.9\%) can reach the nearest hospital in $15 \mathrm{~min}$, however, which only occurs in $9.7 \%$ of the area. The largest percentage of area is $32.5 \%$ which is in 30 to 60 period with only $10.4 \%$ of the population. In the timeframe of more than $60 \mathrm{~min}$, it shows that percentage of area is 6-7 times of the percentage of population. Taking Lanzhou city as a whole, primary medical services can satisfy the demand from $78 \%$ of population, which can obtain medical treatment in $15 \mathrm{~min}$. But $2.4 \%$ of the population (almost 100,000 people) need more than $60 \mathrm{~min}$ to reach primary health care. In terms of hospitals, nearly $85 \%$ of the population can obtain medical treatment within $30 \mathrm{~min}$, but there are still a small number of residents that spend too much time to access medical treatment. Additionally, $1.3 \%$ of the population in $7.5 \%$ of the area spend more than two hours (120 $\mathrm{min}$ ) to reach a hospital. As illustrated in Figure 2, most of the regions with a long medical treatment time are concentrated in the mountainous area in south of $Y D$, the west region of $Y D$, and the north and west regions of $Y Z$, in which there are serious problems such as low road density and scarce medical resources. 
Table 3. Mean time of cost, population, and area within various service range to the nearest medical institution.

\begin{tabular}{|c|c|c|c|c|}
\hline Medical Institution & $\begin{array}{l}\text { Mean Time of } \\
\text { Cost (Min) }\end{array}$ & $\begin{array}{l}\text { Cost Time } \\
\text { (Min) }\end{array}$ & $\begin{array}{l}\text { Pop (Percentage of } \\
\text { Lanzhou City) }\end{array}$ & $\begin{array}{l}\text { Area (Percentage of } \\
\text { Lanzhou City) }\end{array}$ \\
\hline \multirow{6}{*}{ Primary health-care } & \multirow{6}{*}{41.37} & $0-15$ & $78 \%$ & $23.2 \%$ \\
\hline & & $15-30$ & $12.3 \%$ & $26.6 \%$ \\
\hline & & $30-60$ & $7.2 \%$ & $32.5 \%$ \\
\hline & & $60-90$ & $1.1 \%$ & $7.9 \%$ \\
\hline & & $90-120$ & $0.2 \%$ & $1.8 \%$ \\
\hline & & $>120$ & $1.2 \%$ & $8 \%$ \\
\hline \multirow{6}{*}{ Hospital } & \multirow{6}{*}{64.64} & $0-15$ & $69.9 \%$ & $9.7 \%$ \\
\hline & & $15-30$ & $12.9 \%$ & $16.3 \%$ \\
\hline & & $30-60$ & $10.4 \%$ & $32.5 \%$ \\
\hline & & $60-90$ & $3.3 \%$ & $18.6 \%$ \\
\hline & & $90-120$ & $2.2 \%$ & $15.4 \%$ \\
\hline & & $>120$ & $1.3 \%$ & $7.5 \%$ \\
\hline
\end{tabular}
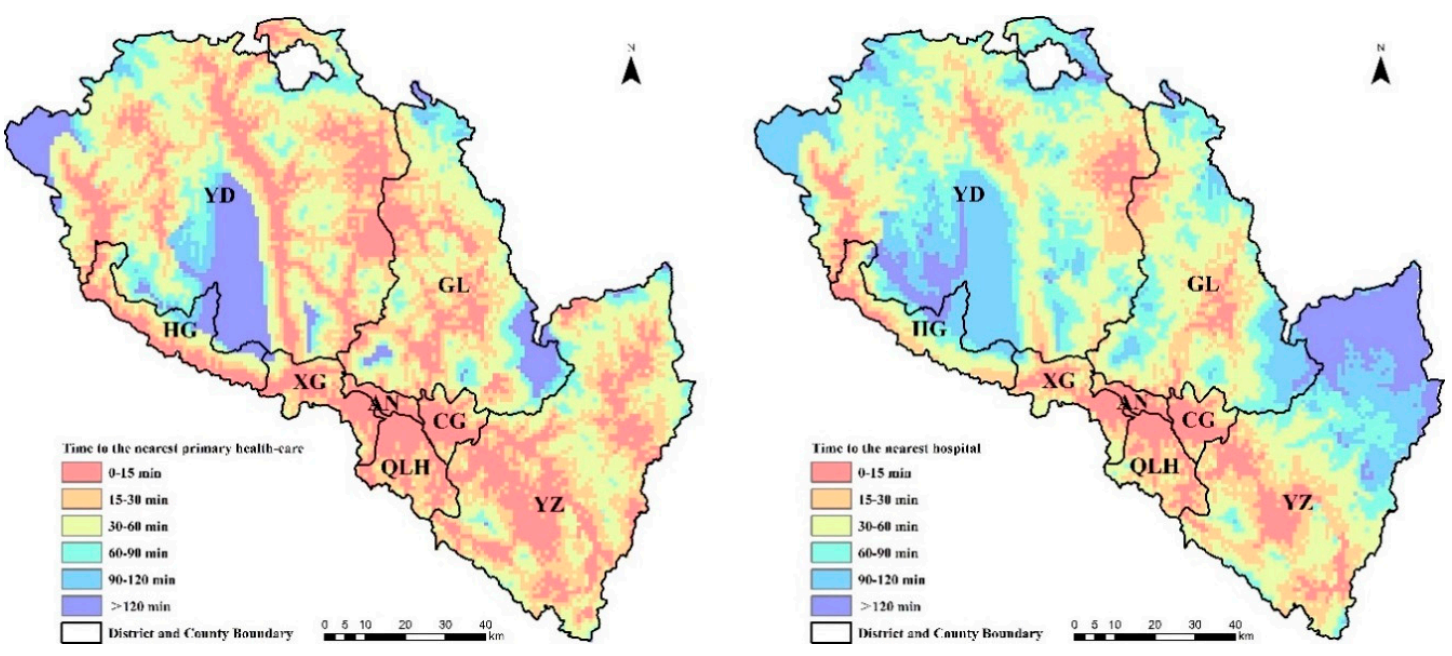

Figure 2. Impedance cost to the nearest medical institution in Lanzhou city.

Accessibility index (Ai) could be calculate by M2SFCA model in analysis of spatial access, which reflects regional difference of accessibility in space. Then disposed Ai data by means of Natural Breaks Classification, dividing it into five levels, respectively, Great, Good, General, Bad, and Very Bad. Referring to Figures 3 and 4, the heterogeneity of spatial accessibility is obvious. The area in grade of Great is less, and areas with accessibility in grade of General and below account for most of the areas in Lanzhou city, so the overall performance of accessibility is poorer. The areas with accessibility in grade of Great and Good distribute locally, while the areas with poorer accessibility scatter in most areas. Accessibility in the central area of YD, the northern area of $Y Z$, and GL is poor relatively, while accessibility is better in HG and other districts. On the whole, spatial accessibility of the north is worse than that of the south, and that of the east and west is worse than that of the middle. Referring to Figure 1, the areas with poor spatial accessibility may be related to the lack of medical resources, the higher terrain in mountainous areas, and the lower coverage degree of road network. 


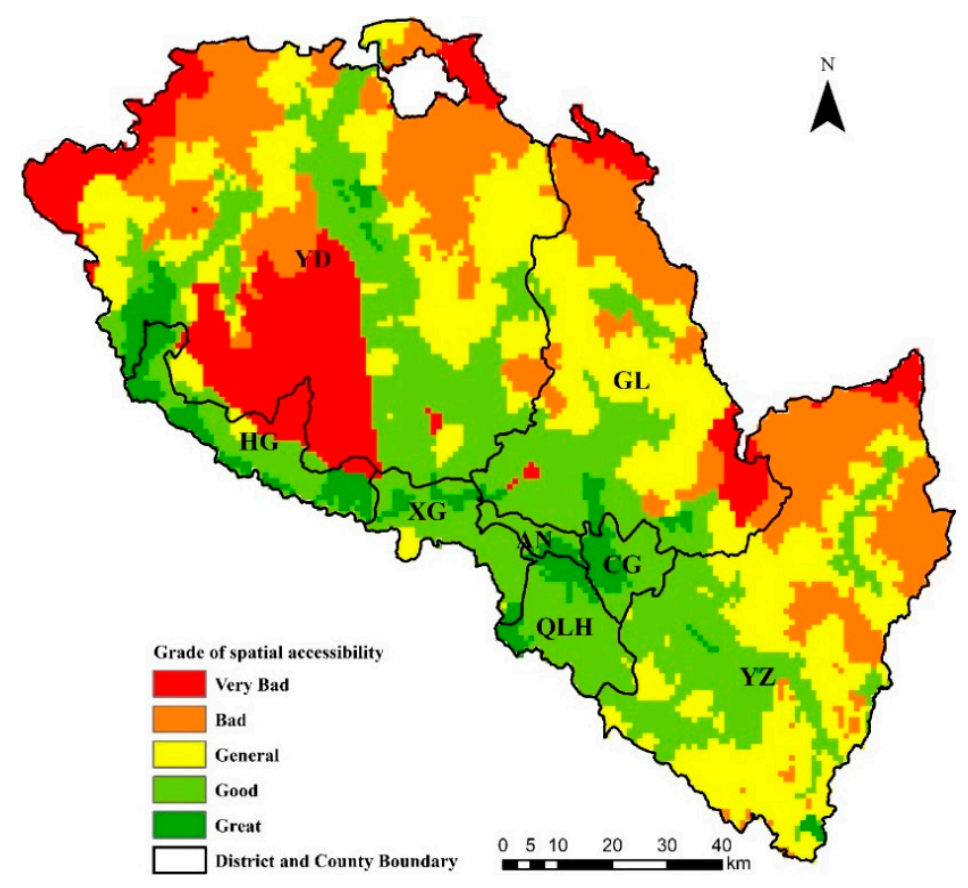

Figure 3. Spatial Accessibility of medical services in Lanzhou city.

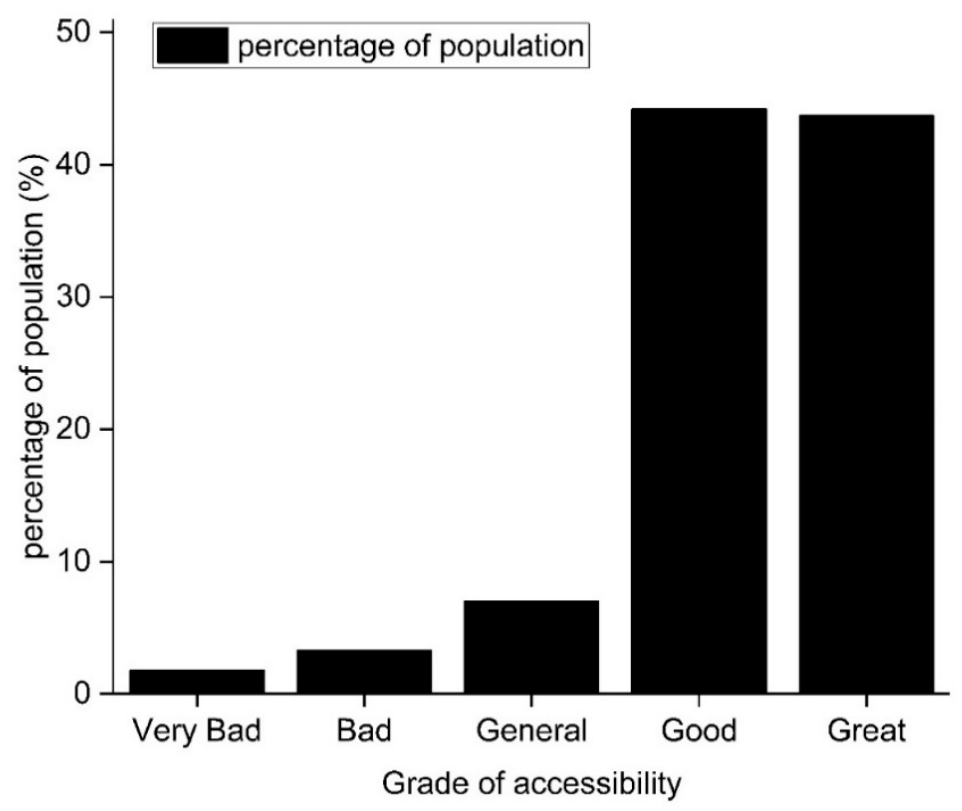

Figure 4. Population proportion in different grade of spatial accessibility.

\subsection{Disparity of Spatial Accessibility Between Urban and Rural Regions}

Referring to the land-use planning of all counties and districts, the urban region and rural region were separated from each of them (Figure 5), and the analysis showed that there is a major difference between the two regions (Table 4). In urban region, areas covered by Ai grade of Great and Good account for more than $90 \%$ of the entire urban region, including $99.9 \%$ of the population in urban region, while proportion of areas and population with accessibility in grade of General and below are $5.5 \%$ and $0.1 \%$, respectively. This indicates that basically all residents in urban region concentrate in the accessibility with better grade. In rural region, $72.5 \%$ of the population distribute within accessibility in better grade, but which only cover $34.3 \%$ of rural region. Nearly $25 \%$ of the population still distribute in areas with accessibility in poorer grade, and the proportion of this area reach up to $65.7 \%$. This 
reveals that the medical and public resources are limited available to most areas in rural region, and there is great zoom for improvement in medical spatial accessibility compared with urban region. From the whole city, it also shows the same rule, that is, most of the population distribute in the areas with better grade of accessibility (Figures 4 and 5). According to the distribution of medical institutions (Figure 1), there are only a few medical institutions in rural region, and the lack of road network in rural region increased the disparities in spatial access between urban and rural regions, which indicates that residents in rural region have unequal opportunities to obtain medical services.

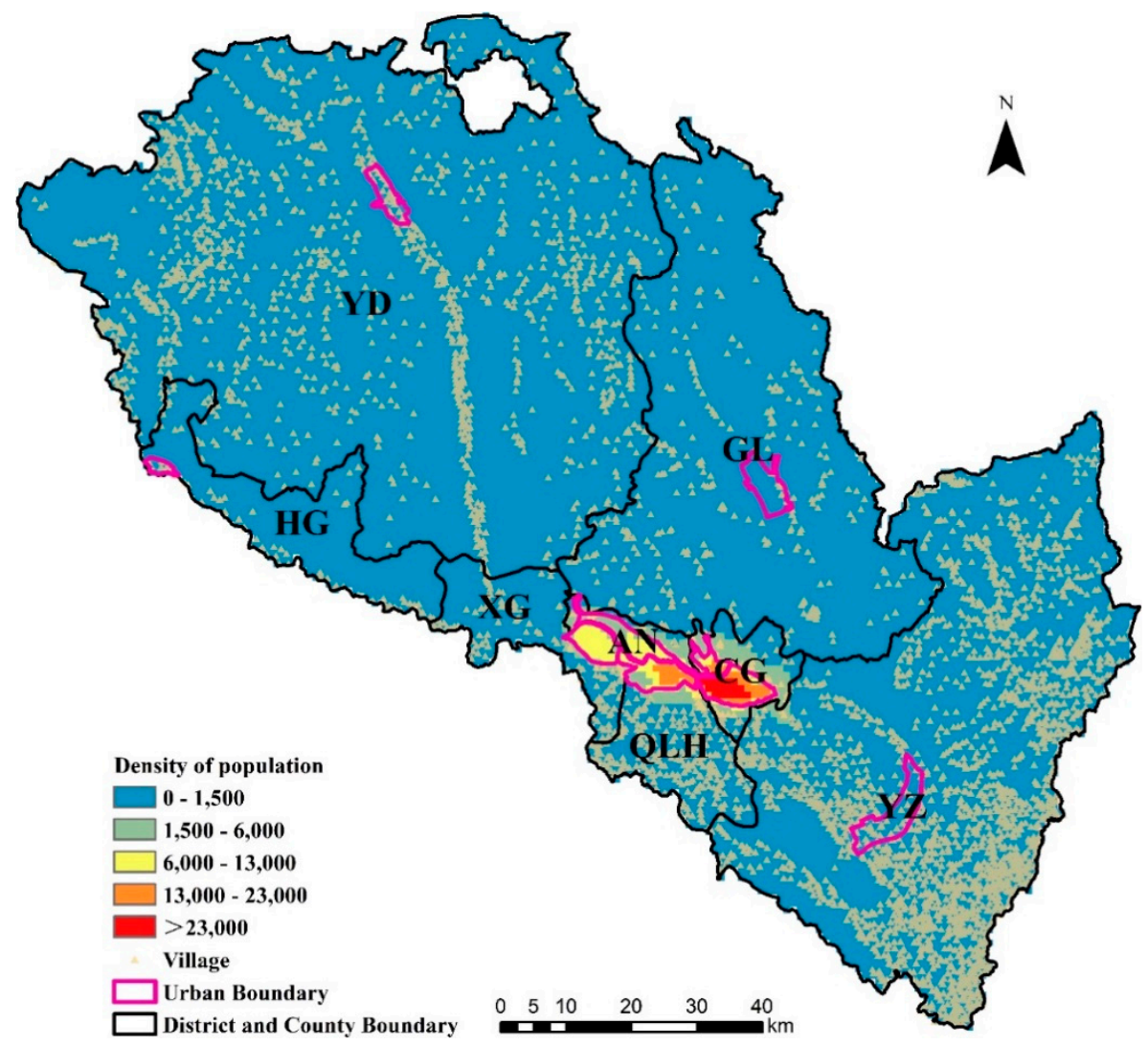

Figure 5. Distribution of population in Lanzhou city.

Table 4. Proportion comparison of Area and population in respective region with accessibility grade between urban and suburban.

\begin{tabular}{ccccc}
\hline Grade & \multicolumn{2}{c}{ Urban } & \multicolumn{2}{c}{ Rural } \\
\hline & Area & Population & Area & Population \\
\hline Great & $32.5 \%$ & $65 \%$ & $4 \%$ & $20.5 \%$ \\
Good & $61.6 \%$ & $34.9 \%$ & $30.5 \%$ & $54.5 \%$ \\
General & $5.9 \%$ & $0.2 \%$ & $29.6 \%$ & $14.4 \%$ \\
Bad & 0 & 0 & $22.4 \%$ & $6.8 \%$ \\
Very Bad & 0 & 0 & $13.5 \%$ & $3.8 \%$ \\
\hline
\end{tabular}

\section{Discussion}

It can be relatively seen from the impedance cost analysis and spatial access analysis: 1 . In primary health care aspect, primary medical services can satisfy the demand from $78 \%$ of the population that can obtain medical treatment in $15 \mathrm{~min}$. In hospital aspect, nearly $85 \%$ of the population can obtain medical treatment within $30 \mathrm{~min}$, but there are still a small number of residents who need to spend too much time to access medical treatment. 2. The spatial distribution pattern of medical accessibility in Lanzhou city is as follows: Areas in parts of main districts, parts of HG, and other tiny minorities 
show Great grade of accessibility, and most of other areas distribute in poorer grade of accessibility. In general, spatial accessibility in north region is worse than that in south region, especially the surrounding mountainous region, such as the central and west parts of $Y D$, the north of $Y Z$, and the junction between GL and YZ. The population mainly distribute in the areas with better grade of accessibility. The inequality of medical treatment among regions is the main reason for the difference in the spatial distribution of medical service accessibility in Lanzhou, and the inequality can be mainly reflected by medical resources and public resources. In daily medical treatment, residents should live in a 15-min health service circle, that is, they can obtain the basic medical resources within 15 min. Therefore, areas that cannot obtain medical services for long travel time should actively strengthen the construction of primary health care. In addition, by observing the location of medical institutions, it can be found that medical resources distribute around the road network. In some areas, such as the central and western mountainous areas of $\mathrm{YD}$, the lack of road network leads to insufficient resources and poor spatial accessibility. There are only township health centers, a few class $0+1$ hospitals and secondary hospitals in the north region of Lanzhou, which leads to the shortage of medical resources and low spatial accessibility of medical treatment in the whole north region, while the high-class hospitals and most medical resources are mainly concentrated in the main districts located in the south region. This is a significant problem for residents with medical needs in other parts of the city. Basically, each town has only one township-level health center stipulated by the state to serve the residents of the whole town, which leads to high accessibility only for those in the immediate area of the health center. This distribution of medical resource allocation is not reasonable, as ideally these resources should be distributed at multiple points within a town. Considering the economic conditions in remote areas, health care workers are not always well paid. Government should improve the compensation of medical staff when carrying out the construction of primary health care facilities, so as to retain talented medical workers in rural and remote areas. Meanwhile, the government should strengthen the professional ethics construction of medical workers who focus on saving lives.

In the analysis of the difference between urban and rural regions in the accessibility of medical services, it can be found that only a small part of the areas shows Great grade of accessibility, but contains the vast majority of the population. In both urban and rural regions, inequality of medical treatment in different degrees is reflected. In urban region, almost all the population (99.9\%) and areas $(94 \%)$ are in the better accessibility grade. The road network and medical resources are relatively sufficient in the urban region of the main districts, and the population distribution is also relatively dense. It indicates that the spatial compatibility among the three elements is decent. However, the over-dense population reduces the per capita acquisition of medical resources, resulting in worse spatial accessibility than expected. Although urban region in other areas do not have such sufficient medical resources and road network, the relatively low population density can also make the spatial compatibility well. Meanwhile, not all urban region can reach the Great grade of accessibility. From the perspective of relevant resource allocation, the major inequality is that a large number of medical resources are concentrated in the east of CG and east of QLH, which leads to over concentrated and saturated distribution of medical resources. In other areas of urban region, such as the west of QLH and the west of AN, medical accessibility has not reached Great grade due to the relative shortage of medical resources. The density of road network is relatively low in some areas, and the lack of resources caused by the low density of road network is also a reason of inequality. In rural areas, the majority of the population is also distributed in the better accessibility grade, but $25 \%$ of the inhabitants covering in $66.5 \%$ of the region are still distributed in the less-than-ideal accessibility grade. Population density in rural region is relatively low. By observing the distribution of $66.5 \%$ of the region in the unsatisfactory grade, it can be found that there are different degrees of shortage of medical resources or road network, which results in comparatively poor spatial compatibility in rural areas. The vast majority of road networks and hospitals are concentrated in areas with a higher grade of accessibility of urban region, which inevitably results in residents (about 25\%) who are located outside not being able to obtain better medical services in the available time. Impedance cost analysis results clearly 
show the problem of unequal resource allocation. However, Great grade areas also exist in rural areas with poor expected accessibility, such as the east and the northwest areas of HG, which indicates that medical resources and public resources in these areas can be fully utilized to meet local needs and achieve a better spatial compatibility and resource allocation. Reasonably allocating the resources, increasing medical resources, and strengthening road network construction are the fundamental means to improve the present worse medical accessibility.

Based on the above, here are the researchers' suggestions for study area: Rural regions should strengthen the construction of hospitals and primary health care, especially the construction of township health centers and health stations, so that health centers and other facilities can be distributed far and near in rural region, rather than only one health center serving an entire town. The medical resources should be distributed in a balanced way as far as possible. High-class hospitals are needed in HG, YD, $\mathrm{YZ}$, and GL, while the medical capacity and facility grade of existing hospitals should be improved to satisfy the demand of local residents. At the same time, Rural areas should strengthen the construction of road networks, especially in the mountainous areas such as the western and central area in YD and the border area between YZ and GL. The publicity that common diseases should be treated in the local primary health care facility while serious diseases in the hospital should be actively promoted in urban region, so as to relieve the pressure from high-grade hospitals.

The research still needs to be further explored in some aspects: Improving the spatial resolution of the analysis grid can reduce the analysis errors in urban areas. Considering the subjective opinions of residents and the medical policies of the government can make the research more realistic. Different real-time road traffic conditions are of great significance to the accessibility under the first-aid mode, and different road network modes (rush hour and non-rush hour) also have a certain impact on the choice of medical treatment. Considering the impact of road network conditions on the accessibility can enhance the practicality of the research.

\section{Conclusions}

The spatial accessibility of medical services in Lanzhou city was analyzed by M2SFCA, and the results show 1. Areas in General and below grade of accessibility account for most of Lanzhou city. Therefore, the spatial accessibility of whole Lanzhou city is comparatively unsatisfactory. It shows a tendency of the north region worse than south region, and areas in parts of the main districts, parts of HG, and other tiny minorities show Great grade of accessibility, and most of the other areas distribute in the poorer grade of accessibility, especially the surrounding mountainous region. 2 . There are obvious differences in accessibility between urban and rural regions, mainly reflected in that residents and areas are basically districted in the Great and Good grade in the urban region, while almost $75 \%$ of the population and $35 \%$ of the areas are distributed in the Great and Good grade, and nearly $25 \%$ of the residents and $65 \%$ of the areas are still in the unsatisfactory accessibility grade in the rural region. According to the results, the researchers put forward corresponding Suggestions to improve the current situation, which are roughly as follows: The construction of hospitals and primary health care should be strengthened in the rural region, especially the construction of township health centers and health stations. High-class hospitals are needed in HG, YD, YZ, and GL, while the medical capacity and facility grade of existing hospitals should be improved to satisfy the demand of local residents. Meanwhile, rural areas should strengthen the construction of road networks.

Author Contributions: Conceptualization, W.X., B.Z. and F.Z.; formal analysis, W.X.; investigation, H.W.; writing-original draft preparation, W.X.; writing—review and editing, F.Z.; supervision, T.Y.

Funding: This research was funded by National Natural Science Foundation of China, grant number 41871075.

Conflicts of Interest: The authors declare no conflict of interest. 


\section{References}

1. Kearns, R.; Moon, G. From medical to health geography: Novelty, place and theory after a decade of change. Prog. Hum. Geogr. 2002, 26, 605-625. [CrossRef]

2. Khan, A.A. An integrated approach to measuring potential spatial access to health care services. Socio-Econ. Plann. Sci. 1992, 26, 275-287. [CrossRef]

3. Luo, W.; Wang, F.H. Measures of spatial accessibility to health care in a GIS environment: Synthesis and a case study in the Chicago region. Environ. Plan. 2003, 30, 865-884. [CrossRef]

4. Ngui, A.N.; Apparicio, P. Optimizing the two-step floating catchment area method for measuring spatial accessibility to medical clinics in Montreal. BMC Health Serv. Res. 2011, 11, 1. [CrossRef] [PubMed]

5. Joseph, A.; Phillips, D. Accessibility and Utilization-Geographical Perspectives on Health Care Delivery; Harper and Row: New York, NY, USA, 1984.

6. McGrail, M.R.; Humphreys, J.S. Measuring spatial accessibility to primary care in rural areas: Improving the effectiveness of the two- step floating catchment area method. Appl. Geogr. 2009, 29, 533-541. [CrossRef]

7. Guagliardo, M.F. Spatial accessibility of primary care: Concepts, methods and challenges. Int. J. Health Geogr. 2004, 3, 3. [CrossRef]

8. Hare, T.S.; Barcus, H. Geographical accessibility and Kentucky's heart-related hospital services. Appl. Geogr. 2007, 27, 181-205. [CrossRef]

9. Luo, J. Integrating the Huff model and floating catchment area methods to analyze spatial access to healthcare services. Trans. GIS 2014, 18, 436-448. [CrossRef]

10. Cheng, G.; Zeng, X.K.; Duan, L.; Lu, X.; Sun, H.; Jiang, T.; Li, Y. Spatial difference analysis for accessibility to high level hospitals based on travel time in Shenzhen, China. Habitat Int. 2016, 53, 485-494. [CrossRef]

11. Guo, J.K.; Qiu, Y.K.; Wang, F.X.; Bai, J.Y. Spatial-Temporal Differences in Accessibility of Pre-Hospital Emergency Medical Services in Dalian based on Traffic Flow. Econ. Geogr. 2019, 39, 82-87.

12. Gao, J.; Tang, S.L.; Rachel, T.; Rao, K.Q. Changing access to health services in urban China: Implications for equity. Health Policy Plan. 2001, 16, 302-312. [CrossRef] [PubMed]

13. Talen, E. School, Community, and Spatial Equity: An Empirical Investigation of Access to Elementary Schools in West Virginia. Ann. Assoc. Am. Geogr. 2001, 91, 465-486. [CrossRef]

14. Song, Z.N.; Chen, W.; Zhang, G.X.; Zhang, L. Spatial accessibility to public service facilities and its measurement approaches. Prog. Geogr. 2010, 29, 1217-1224.

15. Wang, X.L.; Yang, H.Z.; Duan, Z.Q.; Pan, J. Spatial accessibility of primary health care in China: A case study in Sichuan Province. Soc Sci Med. 2018, 209, 14-24. [CrossRef] [PubMed]

16. Chen, J.G.; Zhou, S.H.; Liu, L.; Xiao, L.; Song, G. Estimating the effect of traffic congestion on accessibility to emergency medical care services: Take Guangzhou as an example. Prog. Geogr. 2016, 35, 431-439.

17. Gao, Y.P.; Li, M.Y. Research of spatial accessibility of medical facility based on modified two-step floating catchment area: A case study of nine districts in Shenyang. Inf. Rec. Mater. 2018, 19, 115-118.

18. Tatem, A. WorldPop, open data for spatial demography. Sci. Data 2017, 4, 170004. [CrossRef]

19. Radke, J.; Mu, L. Spatial decompositions, modeling and mapping service regions to predict access to social programs. Geogr. Inf. Sci. A J. Assoc. Chin. Prof. Geogr. Inf. Syst. 2000, 6, 105-112. [CrossRef]

20. Delamater, P.L. Spatial accessibility in suboptimally configured health care systems: A modified two-step floating catchment area (M2SFCA) metric. Health Place 2013, 24, 30-43. [CrossRef]

21. Tao, Z.L.; Cheng, Y. Research progress of the two-step floating catchment area method and extensions. Prog. Geogr. 2016, 35, 589-599.

22. Dai, D.J. Black residential segregation, disparities in spatial access to health care facilities, and late-stage breast cancer diagnosis in metropolitan Detroit. Health Place. 2010, 16(5), 1038-1052. [CrossRef] [PubMed]

23. Luo, W.; Whippo, T. Variable catchment sizes for the twostep floating catchment area (2SFCA) method. Health Place 2012, 18, 789-795. [CrossRef] [PubMed]

24. McGrail, M.R. Spatial accessibility of primary healthcare utilising the two step floating catchment area method: An assessment of recent improvements. Int. J. Health Geogr. 2012, 11(1), 50. [CrossRef] [PubMed]

25. McGrail, M.R.; Humphreys, J.S. Measuring spatial accessibility to primary health care services: Utilising dynamic catchment sizes. Appl. Geogr. 2014, 54, 182-188. [CrossRef] 
26. Tao, Z.L.; Cheng, Y.; Dai, T.Q.; Rosenberg, M.W. Spatial optimization of residential care facility locations in Beijing, China: Maximum equity in accessibility. Int. J. Health Geogr. 2014, 13(1), 33. [CrossRef] [PubMed]

27. Jamtsho, S.; Corner, R.; Dewan, A. Spatio-temporal analysis of spatial accessibility to primary health care in Bhutan. ISPRS Int. J. Geo-Inf. 2015, 4, 1584-1604. [CrossRef]

(C) 2019 by the authors. Licensee MDPI, Basel, Switzerland. This article is an open access article distributed under the terms and conditions of the Creative Commons Attribution (CC BY) license (http://creativecommons.org/licenses/by/4.0/). 